\title{
Perception and Socio-cultural Barriers to the Acceptance of Caesarean Delivery in A Tertiary Hospital in Abakaliki, South East Nigeria
}

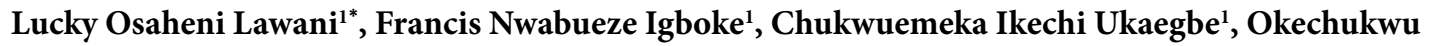 \\ Bonaventure Anozie ${ }^{1}$, Chukwuemeka Anthony Iyoke ${ }^{2}$, Fidelis Agwu Onu${ }^{1}$, Tagbo Anthony Agbata ${ }^{1}$, \\ Obiora Asiegbu ${ }^{1}$
}

\begin{abstract}
Objectives: Globally, caesarean section (CS) has immensely contributed to improved obstetric outcome in circumstances where vaginal delivery is not feasible. However, in some low-income countries, there is aversion to the procedure. The aim of this study was to determine the level of awareness and socio-cultural barriers to the acceptance of CS.

Materials and Methods: A cross-sectional study was conducted among 344 parturients at Federal Teaching Hospital in Abakaliki, from October 1 to November 30, 2016. Data were collated using a self-administered questionnaire and was analyzed using SPSS version 20.0 and conclusions were drawn by means of descriptive statistics.

Results: All the respondents were aware of CS as an operative abdominal procedure for delivery; of these, over one-tenth (14.0\%; $48 / 344)$ had experienced the procedure previously. Over four-fifths $(82.3 \% ; 283 / 344)$ of those who have had a previous CS were wellinformed about the indications. About one-fifth (20.3\%; 70/344) did not accept CS for any reasons. The major barriers to acceptance were being considered by peers as a reproductive failure $(29.2 \% ; 7 / 24)$, high cost $(20.8 \% ; 5 / 24)$ and religious beliefs $(12.5 \%$; 3/24). Conclusions: Majority of antenatal attendees had a significant awareness of CS and the indications. Moreover, a vast majority had morbid aversion towards it; due to numerous, non-evidence based socio-cultural reasons. Therefore, adequate health education, female empowerment, access to free or affordable antenatal care service, elimination of harmful, religious/cultural beliefs and myth regarding caesarean delivery are necessary to curb this ugly trend if we hope to achieve the sustainable development goals related to maternal health.

Keywords: Perception, Acceptance, Barriers, Caesarean section, Abakaliki, Nigeria
\end{abstract}

\section{Introduction}

There is no greater joy than the birth of a healthy baby and no greater tragedy than the death of a mother and her baby following a complicated pregnancy or difficult labor, especially when it occurs due to the refusal of caesarean birth. As often as one thousand times a day, women die while giving birth. Many of which occur due to the refusal of caesarean delivery in some low resource settings $(1,2)$. Caesarean section (CS) as an operative technique has contributed immensely to improved obstetric care throughout the world (1.2). It serves as a surgical procedure facilitating rapid delivery of the baby when prolongation of the pregnancy and/or labor is deemed undesirable and the vaginal route is not feasible (3).

The incidence of CS in most health institutions in Nigeria ranges between 20 and 30\% (4), but globally, it is about $10 \%$ to $35 \%$ (5). Current improvements in the technique of CS, safer anesthesia, newer and potent antibiotics and availability of blood transfusion services have now made CS safer. In addition, with better education and enlightenment, it is becoming more acceptable to women and their families (6). Due to the current safety of the procedure, several CS are done for various justifiable medical and non-medical indications with favorable outcomes $(7,8)$. These may have contributed a lot to the increased rate of the procedure in both low and highincome countries of the world.

However, in some low-income countries like Nigeria, many women and their families still have numerous negative perceptions regarding caesarean delivery. In these settings, women who had caesarean delivery were considered as weaklings and a reproductive failure (6). Failure to deliver vaginally may be attributed to a curse on an unfaithful woman $(1,6)$. Rather, vaginal delivery in such settings is considered as the proof of womanhood $(9,10)$. Other reasons adduced for the aversion to CS by women in developing countries include the morbidity and mortality from the procedure, prolonged hospital stay and perceived high cost of hospital bills.

A large number of people in low-income countries 
still hold strong negative cultural perceptions regarding caesarean delivery, despite the availability of evidencebased safe techniques and improvements. Adeoye et al in 2011 reported that $34 \%$ of respondents stated that the cultural influence of their communities was responsible for their negative perception of abdominal delivery (1). Moreover, Aziken et al in 2007 reported that 1.8\% of women rejected caesarean delivery because it was not acceptable by their culture (11). Orji et al (12) and Bello et al (13) documented that these cultural reasons also include the fact that caesarean delivery was felt to be due to spiritual attacks, retribution for women's infidelity and failure of a woman to fulfill her reproductive functions.

Fear of death during or after the procedure is another significant reason why many women will refuse to have a caesarean delivery. Chigbu and Iloabachie in 2007 stated that reasons underlying these fears were death of close relatives during CS, past unpleasant experiences in previous caesarean deliveries and unpleasant stories that they heard from other women (14).

The financial implication of the procedure is another reason why women refused it. Ezechi et al in 2004 reported that $66.5 \%$ of respondents in their study declined caesarean delivery due to the high cost of the procedure, especially in settings without functional health insurance schemes (15). Chigbu et al in 2007 recognized the high cost of CS as the basis for refusing the procedure. In low-income settings like ours, low minimum wage, poor implementation of the National Health Insurance Scheme and low uptake of family planning practices combine to inflict more economic pressures on households leaving little or nothing for proper health maintenance (11).

Jayleen et al in 2017 revealed that contrary to the increasing trend in the use of CS in low-income countries, women in Enugu, southeastern Nigeria, had limited access to CS. Increasing age and socioeconomic proxies for income and access to care were shown to be key determinants of access to CS. They, therefore, recommended further research to ascertain the obstetric conditions under which women in this region receive CS, and to further elucidate the role of socioeconomic factors in accessing CS (16). This necessitated the current study which aimed to assess awareness, acceptance of caesarean delivery, and reasons for possible aversion/determinants of access to CS in a tertiary health institution in Abakaliki, southeastern Nigerian, with a view to providing local and regional data which will be helpful in designing, promoting and implementing health interventions and policies aimed at optimizing the care of women and improving the outcome of pregnancy.

\section{Materials and Methods}

This cross-sectional study included pregnant women attending the antenatal clinic at the Federal Teaching Hospital Abakaliki (FETHA). All consenting consecutive booked client who attended the clinic from October 1 to
November 30, 2016, were approached to participate in the study. The essence of the study, consent form and relevant sections of the questionnaires were fully explained by the principal researcher and the research assistants. Thereafter, written consent was obtained and participants were interviewed using a structured self-administered questionnaire. The questionnaire was structured to collect information on the respondents' socio-demographic variables like age, parity, gestational age, level of education and occupation, as well as awareness of CS, their perception of the procedure, aversion and reasons for aversion.

The study sample size of 360 was derived based on the formula: $\mathrm{N}=\mathrm{z}^{2} \mathrm{pq} / \mathrm{e}^{2}$, where $\mathrm{N}=$ minimum required sample size, $\mathrm{Z}=$ standard variate (1.96), $\mathrm{P}=$ estimated prevalence (0.307) (obtained as the current CS rate (30.7\%) (17) in FETHA, Q = (1-p), $\mathrm{E}^{2}=$ acceptable error at 0.05 $\left(\mathrm{N}=(1.96)^{2}(0.307)(0.693) /(0.05)^{2}=327\right)$. The minimum sample size was further increased by $10 \%$ attrition value (32.7). The total sample size was $327+32.7=360$. However, 16 participants were excluded from the study due to incorrectly filled questionnaires, giving a total of 344 eligible respondents. Data were collated and analyzed using Statistical Package for Social Science (SPSS) version 20.0 and conclusions were drawn by means of descriptive statistics.

\section{Results}

A total of 344 questionnaires were suitable for analysis, giving a response rate of $95.6 \%$. Two-fifths of the respondents $(138 / 344 ; 40.1 \%)$ were within the age range of 26-30 years. Over half $(206 / 344 ; 59.9 \%)$ of respondents were in the third trimester of their pregnancy. The parity and other socio-demographic characteristics of the respondents are shown in Table 1.

All the respondents were aware of CS as an obstetric procedure, with about one-third, each stating that it is performed for the benefit of the baby $(126 / 344 ; 36.3 \%)$ and mother $(112 / 344 ; 32.6 \%)$ respectively, while over one-tenth $(45 / 344 ; 13.1 \%)$ was of the opinion that it performed for the benefits of both the baby and mother. Unfortunately, $1.5 \%$ of respondents who had awareness did not know the indication for the procedure. The other indications enumerated by other respondents are shown in Table 2.

Amongst the respondents, $14.0 \%$ (48/344) have had a previous CS, while over four-fifths $(296 / 344 ; 86.0 \%)$ had not. The various indications for which the procedure was performed are shown in Table 3 , indicating that abnormal presentations $(8 / 48 ; 16.6 \%)$ and fetal distress $(8 / 48 ; 16.6 \%)$ were the major indications for the procedure. Surprisingly, $6.3 \%(3 / 48)$ of the respondents were not told the reason(s) for performing a CS on them.

Majorities $(94.5 \% ; 320 / 344)$ of the patients have never declined a caesarean delivery, while $5.5 \%$; 24/344 had done so previously. The reasons adduced for refusal were mainly that they did not want to be seen by their peers and 


\begin{tabular}{lc}
\multicolumn{2}{l}{ Table 1. Socio-demographic Characteristics of Participants (N=344) } \\
\hline Variables & No. (\%) \\
\hline Age (y) & $13(3.8)$ \\
$\leq 20$ & $88(25.6)$ \\
$21-25$ & $138(40.1)$ \\
$26-30$ & $81(23.5)$ \\
$31-35$ & $24(7.0)$ \\
$>35$ & \\
Educational level & $2(0.6)$ \\
No formal education & $13(3.8)$ \\
Primary & $88(25.6)$ \\
Secondary & $241(70.0)$ \\
Tertiary & \\
Parity & $104(30.2)$ \\
0 & $221(64.3)$ \\
$1-4$ & $19(5.5)$ \\
$\geq 5$ & \\
Gestational age (wk) & $31(9.0)$ \\
$1-13$ & $107(31.1)$ \\
$14-28$ & $206(59.9)$ \\
$>28$ & \\
Occupation & $48(14.0)$ \\
Professional & $190(55.2)$ \\
Skilled & $60(17.4)$ \\
Unskilled & $46(13.4)$ \\
Unemployed & \\
\hline & \\
&
\end{tabular}

Table 2. Awareness of Caesarean Section

\begin{tabular}{lc}
\hline Variables & No. (\%) \\
\hline Have you heard of Caesarean section? & $344(100.0)$ \\
Yes & $0(0.0)$ \\
No & \\
Why do you think Caesarean section is performed? & $126(36.6)$ \\
Benefit of baby & $112(32.6)$ \\
Benefit of the mother & $45(13.1)$ \\
Benefit of both the mother and baby & $8(2.3)$ \\
Convenience of the doctor & $48(13.9)$ \\
Financial benefit to care provider & $5(1.5)$ \\
No idea &
\end{tabular}

neighbors as weaklings or a reproductive failure (29.2\%; $7 / 24)$, the high cost of the procedure $(20.8 \% ; 5 / 24)$, religious belief $(12.5 \% ; 3 / 24)$, fear of future caesarean delivery $(8.3 \% ; 2 / 24)$, desire for large family size $(4.2 \%$; $1 / 24$ ), other reasons are shown in Table 4.

When asked if they would accept a CS when indicated in future, $79.7 \%$ (274/344) said they would accept, while the remainder said they would decline. However, the reasons given for future refusal were similar to but of varying proportions to the reasons given by those who previously declined the procedure (Table 4).

Evaluation of the perception and outcome regarding
Table 3. History of Previous Caesarean Section and its Indication

\begin{tabular}{lc}
\hline Variables & No. (\%) \\
\hline Have you had a previous caesarean section? & $48(14.0)$ \\
Yes & $296(86.0)$ \\
No & \\
Indication for previous caesarean section, N=48 & $8(16.6)$ \\
Abnormal presentation & $3(6.3)$ \\
Abnormal lie & $8(16.6)$ \\
Fetal macrosomia & $3(6.3)$ \\
Fetal distress & $5(10.4)$ \\
Obstructed labor & $2(4.3)$ \\
Inadequate pelvis & $5(10.4)$ \\
Maternal medical conditions & $1(2.0)$ \\
Placenta praevia & $2(4.2)$ \\
Postdate pregnancy & $1(2.0)$ \\
Previous scar & $7(14.6)$ \\
Prolonged labor & $3(6.3)$ \\
Not informed &
\end{tabular}

CS, Caesarean section.

the mode of delivery shows that $11.6 \%(40 / 344)$ believed that they can have a successful vaginal delivery even against medical recommendation. A small proportion $(1.2 \% ; 3 / 240)$ of those who were told they cannot deliver vaginally except through CS said they managed to deliver vaginally, while the majority $(98.8 \% ; 237 / 240)$ had caesarean delivery (Table 5).

\section{Discussion}

The aversion to caesarean delivery among women in Nigeria was previously documented by Chigbu and Iloabachie in 2007 (14) and Ezechi in 2004 (15) respectively. Unfortunately, after more than a decade of this finding, the results of the current study are still consistent with those of these researchers, indicating that barriers to the acceptance of CS still exist. The attitude of Nigerian women towards CS in the current study was influenced by socio-cultural factors, religious beliefs and economic reasons, a finding which is in tandem with previous reports $(2,16)$.

Approximately two-fifths (40.1\%) of the respondents were within the age range of 26-30 years. This is expectedly so as the subjects were mainly women of reproductive age. This is similar to the findings of other researchers who reported that this was the commonest age group of women seen in antenatal clinics $(2,18)$. In addition, about two-thirds $(64.2 \%)$ of respondents in this study were primiparae and multiparae combined and $59.9 \%$ were in the third trimester. This is important because parity and the greater number of antenatal visits have been shown to affect one's decisions or re-shape already held views concerning the mode of delivery $(19,20)$.

Despite the fact that more than four-fifths of the respondents had secondary and tertiary formal education, the majority continued to have aversion to caesarean 
Table 4. Previously Declined Caesarean Section and Reasons for Refusing

\begin{tabular}{|c|c|}
\hline Variables & No. (\%) \\
\hline \multicolumn{2}{|l|}{ Have you ever refused Caesarean delivery? } \\
\hline No & $320(94.5)$ \\
\hline Yes & $24(5.5)$ \\
\hline \multicolumn{2}{|l|}{ Reasons for previous refusal, $n=24$} \\
\hline Seen as reproductive failure & $7(29.2)$ \\
\hline High cost & $5(20.8)$ \\
\hline Fear of future caesarean sections & $2(8.3)$ \\
\hline Religious belief & $3(12.5)$ \\
\hline Desire for large family size & $1(4.2)$ \\
\hline Death of a relative from CS & $1(4.2)$ \\
\hline Fear of death & $2(8.3)$ \\
\hline Partners' and family members' decision & $2(8.3)$ \\
\hline No reasons & $1(4.2)$ \\
\hline \multicolumn{2}{|c|}{ Will you refuse a caesarean section in the future? } \\
\hline No & $274(79.7)$ \\
\hline Yes & $70(20.3)$ \\
\hline \multicolumn{2}{|l|}{ Reasons for future refusal, $n=70$} \\
\hline Seen as reproductive failure & $17(24.3)$ \\
\hline High cost & $7(10.0)$ \\
\hline Religious belief & 9 (12.9) \\
\hline Death of a relative from CS & $1(1.4)$ \\
\hline Inadequate counseling by doctors & $3(4.3)$ \\
\hline Fear of death & $15(21.4)$ \\
\hline Would not accept for no reason(s) & $18(25.7)$ \\
\hline
\end{tabular}

CS, Caesarean section.

Table 5. Perception and Outcome Regarding Route/Mode of Delivery Opted for Against Medical Advice

\begin{tabular}{lc}
\hline Variables & No. (\%) \\
\hline $\begin{array}{l}\text { Do you think you can have a successful vaginal delivery } \\
\text { even against medical recommendation? }\end{array}$ \\
No & $304(88.4)$ \\
Yes & $40(11.6)$ \\
& \\
Have you ever been told that you cannot deliver & \\
vaginally, but later you delivered? N=240 & \\
No & $237(98.8)$ \\
Yes & $3(1.2)$ \\
\hline
\end{tabular}

delivery even in the presence of obstetric indications. This finding is at variance with those of other researchers in some parts of Nigeria, India and Brazil $(11,18,19)$, where education was found to economically empower women and their families and therefore made them more favorably disposed to have an improved health seeking behavior and less likely to refuse CS for economic reasons (14). However, in the present study, education did not dispel major militating socio-cultural factors affecting either acceptance or rejection of the procedure. Similarly, in Lagos, Nigeria, Ezechi et al reported that education and social class had little or no effect on aversion to CS (15).
Hopefully, proper health education and counseling are expected to positively influence the attitude of parturient, as seen in high-income countries, with the prospect of translating such gains into better health-seeking behavior and outcome.

However, Jayleen et al in Enugu, southeastern Nigeria, were of the opinion that in order to meet the Sustainable Development Goal and decrease maternal mortality, increased access to CS as an obstetric intervention is of critical importance (16). They reported in their analysis that women in this region had limited access to caesarean delivery compared to the increasing global trend. Furthermore, similar to the current study, they found that socioeconomic variables like education, employment status and residence were key determinants of access to caesarean birth (16). Despite both Enugu and Abakaliki being urban cities, the current study shows that women referred to FETHA, Abakailki, were less willing to take up the intervention that their urban counterparts in Enugu who had better access (16). This may be due to the fact that FETHA serves as a referral center, for most patients referred with lower levels of healthcare from private, primary and secondary health care facilities located in rural areas.

The current study showed that all the respondents have heard about CS, with the majority of the respondents exhibiting correct awareness of the indications for CS as an operative procedure used for delivery. This may be attributed to their booking status and counselling. This finding is consistent with that of Adageba in Ghana, in which respondents showed an increased awareness of CS (21). However, of the one-tenth (14.0\%) who have had a previous CS, 93.7\% were aware of the indications, while $6.3 \%$ had no idea of the indication(s) for the procedure. This again could be attributed to poor counseling and lack of health education in their previous pregnancies, a finding which is consistent with that of Ugwu et al in Enugu, Nigeria (22).

Majority of the antenatal attendees have never refused caesarean delivery, possibly because they have either not been confronted with such a situation or only a small fraction has been, however, $5.5 \%$ of them had previously declined caesarean delivery. This is less than the $12 \%$ reported by Chigbu and Iloabachie (14) in Enugu, Nigeria. These women declined the procedure for a number of socio-cultural reasons similar to those adduced by the respondents in the current study. The reasons attributed for refusal in this study were mainly due to wrong cultural beliefs and myth, fear of being perceived and viewed as a reproductive failure, fear of death from unsafe procedure, and high cost of the procedure in the absence of health insurance necessitating out-of-pocket health financing in a setting with high poverty rate. These reasons are similar to those reported by Aziken et al (11), in which 19\% would not accept the procedure, and also with the $13.4 \%$ 
and $12.1 \%$ reported by Adeoye et al (1) and Orji et al (12) respectively.

When asked if they would accept CS for future deliveries, almost four-fifths were willing to accept as long as there are indications, while about one-fifth was unwilling to do so, even in the presence of medical or obstetric indications. The reasons for refusal were similar to those given by those who had declined CSs in their past confinements. These findings were in tandem with earlier reports by Aziken et al, Adeoye\& Kalu and Orji et al respectively $(1,11,12)$. Contrary to the finding of the present study, $55 \%$ of women in Ghana who had a previous CS preferred vaginal delivery over a repeat CS (23).

In most Nigerian setting, some women will opt for or insist on vaginal delivery even when contra-indicated and despite repeated counseling on the potential danger of such action. It is therefore not surprising that $11.6 \%$ of the study subject believe they will have a successful vaginal delivery when medically contraindicated and despite the advice of a doctor. This finding is similar to those of Jeremiah et al and Orji et al $(2,12)$.

In conclusion, this study found that the majority of the women who accessed antenatal care services in FETHA during the study period had a high awareness of CS and its indications. However, a vast majority had morbid aversion towards the procedure due to numerous, non-evidence based socio-cultural reasons, poverty and illiteracy. For CS to become more widely accepted by parturient in our setting, we advocate that women should receive adequate counseling and health education, be enlightened, become empowered, have access to free or affordable antenatal care service and abolition of out-of-pocket payment of the fee for service system of health financing. Religious and traditional leaders also have a significant and massive role to play in correcting wrong religious and cultural beliefs and myth regarding caesarean delivery if we sincerely hope to achieve the sustainable development goals related to maternal health.

\section{Conflict of Interests}

Authors declare that they have no conflict of interests.

\section{Ethical Issues}

Ethical approval for the study was obtained from the Research Ethics Committee of the hospital (REC protocol number: 23/01/2014-07/02/2014; REC approval number: 07/02/2014-24/04/2014).

\section{Financial Support}

No funding received.

\section{References}

1. Sunday-Adeoye I, Kalu CA. Pregnant Nigerian women's view of cesarean section. Niger J Clin Pract. 2011;14(3):276279. doi:10.4103/1119-3077.86766

2. Jeremiah I, Nonye-Enyidah E, Fiebai P. Attitudes of antenatal patients at a tertiary hospital in Southern Nigeria towards caesarean section. J Public Health Epidemiol. 2011;3(13):617-621. doi:10.5897/JPHE11.22

3. Inyang-Etoh EC, Etuk SJ. Demographic and obstetric determinants of emergency caesarean section among women in Calabar, Nigeria. Global Research Journal of Medical Sciences. 2013;3(1):20-24.

4. Ebeigbe PN, Ilesami AO. Caesarean Delivery. In: Okpere E, eds. Clinical Obstetrics (Revised Edition). Benin City: University of Benin Press; 2004:337-343.

5. Nwobodo EI, Isah AY, Panti A. Elective caesarean section in a tertiary hospital in Sokoto, north western Nigeria. Niger Med J. 2011;52(4):263-265. doi:10.4103/0300-1652.93801

6. Ojiyi E, Anolue F, Dike E, Chukwulebe A. Appraisal of Caesarean Section at the Imo State University Teaching Hospital, Orlu, South-eastern Nigeria. The Internet Journal of Gynecology and Obstetrics. 2012;16(2).

7. Penna L, Arulkumaran S. Cesarean section for non-medical reasons. Int J Gynaecol Obstet. 2003;82(3):399-409.

8. Finger C. Caesarean section rates skyrocket in Brazil. Many women are opting for caesareans in the belief that it is a practical solution. Lancet. 2003;362(9384):628. doi:10.1016/ s0140-6736(03)14204-3

9. Ezechi OC, Nwokoro CA, Kalu BKE, Njokanma FO, Okeke GCE. Caesarean morbidity and mortality in a private hospital in Lagos, Nigeria. Trop J Obstet Gynaecol. 2002;19(2):97-100.

10. Ezechi OC, Edet A, Akinlade H, Gab-Okafor CV, Herbertson E. Incidence and risk factors for caesarean wound infection in Lagos Nigeria. BMC Res Notes. 2009;2:186. doi:10.1186/1756-0500-2-186

11. Aziken M, Omo-Aghoja L, Okonofua F. Perceptions and attitudes of pregnant women towards caesarean section in urban Nigeria. Acta Obstet Gynecol Scand. 2007;86(1):4247. doi:10.1080/00016340600994950

12. Orji EO, Ogunniyi SO, Onwudiegwu U. Beliefs and perceptions of pregnant women at Ileşa about caesarean section. Trop J Obstet Gynaecol. 2003;20(2):141-143.

13. Bello FA, Olayemi O, Ogunbode OO, Adekunle AO. Attitude to caesarean section amongst antenatal clients in Ibadan, Nigeria. Trop J Health Sci. 2011;18(1). doi:10.4314/ tjhc.v18i1.64483

14. Chigbu CO, Iloabachie GC. The burden of caesarean section refusal in a developing country setting. BJOG. 2007;114(10):1261-1265. doi:10.1111/j.14710528.2007.01440.x

15. Ezechi CO, Fasubaa OB, Kalu BEK, Nwokoro CA, Obiesie LO. Caesarean delivery: why the aversion? Trop J Obstet Gynaecol. 2004;21(2):164-167. doi:10.4314/tjog. v21i2.14494

16. Gunn JK, Ehiri JE, Jacobs ET, et al. Prevalence of Caesarean sections in Enugu, southeast Nigeria: Analysis of data from the Healthy Beginning Initiative. PLoS One. 2017;12(3):e0174369. doi:10.1371/journal.pone.0174369

17. Agboeze J, Onoh RC, Umeora OUJ, et al. Microbiological pattern of postcesarean wound infection at Federal Teaching Hospital, Abakaliki. Afr J Med Health Sci. 2013;12(2):99102. doi:10.4103/2384-5589.134905

18. Allagoa DO, Nyengidiki TK. Knowledge, Attitude and Practice of Contraception Amongst Antenatal Patients at The University of Port Harcourt Teaching Hospital, Port 
Harcourt. Nigerian Health Journal. 2011;11(3):89-92.

19. Niathani U, Bhawal P, Chauhau SS, Kumar D, Gupta S. Knowledge, attitude and acceptance of antenatal women toward labor analgesia and caesarean section in a medical college hospital in India. J Obstet Anaesth Crit Care. 2011;1(1):13-20. doi:10.4103/2249-4472.84250

20. Ribeiro VS, Figueiredo FP, Silva AA, et al. Why are the rates of cesarean section in Brazil higher in more developed cities than in less developed ones? Braz J Med Biol Res. 2007;40(9):1211-1220.

21. Adageba R, Danso K, Adusu-Donkor A, Ankobea-Kokroe
F. Awareness and Perceptions of and Attitudes towards Caesarean Delivery among Antenatal. Ghana Med J. 2008;42(4):137-140.

22. Ugwu GO, Iyoke CA, Onah HE, Egwuatu VE, Ezugwu FO. Maternal and perinatal outcomes of delivery after a previous Cesarean section in Enugu, Southeast Nigeria: a prospective observational study. Int J Womens Health. 2014;6:301-305. doi:10.2147/ijwh.s56147

23. Danso K, Schwandt H, Turpin C, Seffah J, Samba A, Hindin M. Preference of ghanaian women for vaginal or caesarean delivery postpartum. Ghana Med J. 2009;43(1):29-33.

(C) 2019 The Author (s); This is an open-access article distributed under the terms of the Creative Commons Attribution License (http://creativecommons.org/licenses/by/4.0), which permits unrestricted use, distribution, and reproduction in any medium, provided the original work is properly cited. 